\title{
Optical Turbulence and Outer Scales above Dome C in Antarctica
}

\author{
Hervé Trinquet $^{a}$, Abdelkrim Agabi $^{a}$, Jean Vernin $^{a}$, Max Azouit $^{a}$, Eric Aristidi $^{a}$, Eric Fossat $^{a}$ \\ ${ }^{a}$ Laboratoire H. Fizeau, Université de Nice Sophia-Antipolis, CNRS, Observatoire de la Côte \\ d'Azur, UMR 6525, Parc Valrose, 06108 Nice, France
}

\begin{abstract}
Dome $\mathrm{C}$ in Antarctica is a particular astronomical site when considering the optical turbulence conditions. From the first winterover campaign performed in 2005 at Dome C, the set of 34 meteorological balloon profiles has been analyzed. The meteorological balloons were equipped with microthermal sensors used to sense the vertical profile of the optical turbulence intensity $C_{n}^{2}$. The $C_{n}^{2}$ median profile, mean temperature and mean horizontal wind speed are given. The $C_{n}^{2}$ median profile is characterized by a very strong and thin turbulent surface layer. The surface layer height is defined. The median outer scale profile at Dome $\mathrm{C}$ is computed using the Tatarski definition. The von Kármán outer scale is also deduced. The integrated parameters as Fried parameter $r_{0}$, coherence time $\tau_{0}$, isoplanatic angle $\theta_{0}$ and the spatial-coherence outer scale $\mathscr{L}_{0}$ used to define astronomical site quality, are computed at $8 \mathrm{~m}$ above the ground and above the turbulent surface layer.
\end{abstract}

Keywords: atmospheric effects, interferometry, site testing, outer scale

\section{INTRODUCTION}

Dome $\mathrm{C}$ on the Antarctic plateau has many advantages for Astronomy. At $3233 \mathrm{~m}$ of altitude, the dry, cold and clear air and with the possibility to observe without discontinuity during several months, placed Dome $\mathrm{C}$ as a potentially interesting astronomical site. Ground based telescopes and high angular resolution technics, like adaptive optics or interferometry, are highly concerned by atmospheric turbulence as it drastically reduce the angular resolution of telescopes.

\section{OPTICAL TURBULENCE}

Since 1960, the effects of atmospheric turbulence on wave propagation, called optical turbulence, has been studied. The turbulent process as suggested by Richardson in $1922,{ }^{1}$ may be described by a flow of energy: a small fraction of the ambient kinetic energy of the flow is converted into turbulence energy. The energy is injected through a large eddy at the size of the outer length scale $L_{0}$ which is then break up into smaller eddies. The input energy is progressively divided and redistributed to smaller and smaller scales. The energy cascade continues until the eddies are comparable in sizes to the inner scale length $l_{0}$, below which the energy is dissipated by viscosity in heat. The inertial range is defined between the energy input region and the energy-loss region $l_{0}<\rho<L_{0}$. In this range, Kolmogorov in $1941^{2}$ assumed that the velocity fluctuations are both isotropic and homogeneous and established the so well known $2 / 3$ scaling law for the fluctuations of the velocity component. The structure function of the velocity is expressed as a function of the distance $\rho$ between two points and $\epsilon$ the energy dissipation rate $\left[\mathrm{m}^{2} \mathrm{~s}^{-3}\right]$ :

$$
D_{v}(\rho)=<(v(r)-v(r+\rho))^{2}>=c(\epsilon \rho)^{2 / 3}=C_{v}^{2} \rho^{2 / 3},
$$

where $c$ is a dimensionless constant and $C_{v}^{2}$ is the velocity structure constant. The same analysis may be applied to describe the turbulent mixing of a conservative and passive scalar as made by Obukhov in $1949^{3}$ on the refractive-index. The refractive-index structure constant $C_{n}^{2}$ is defined by:

$$
D_{n}(\rho)=C_{n}^{2} \rho^{2 / 3},
$$

and $C_{n}^{2}$ is the relevant parameter which quantify the optical turbulence strength. 
The refractive index of air is a function of the absolute temperature $T$, the atmospheric pressure $p$ and the partial pressure of water vapor $e$ :

$$
n=1+\frac{79.10^{-6}}{T}\left(p+\frac{4800 e}{T}\right)
$$

Since $T$ is not a conservative and passive scalar, the potential temperature $\theta$ has to be considered:

$$
\theta=T\left(\frac{1000}{P}\right)^{0.286}
$$

which is the temperature of parcels of air displaced adiabatically at $1000 \mathrm{Hpa}$. Like in most of astronomical site the effect of the humidity may be neglected and the index structure constant $C_{n}^{2}$ is related to the temperature structure constant $C_{\theta}^{2}$ according to the Gladstone formula:

$$
C_{n}^{2}=C_{\theta}^{2}\left(\frac{79.10^{-6} P}{T^{2}}\right)^{2}
$$

\section{3. $C_{N}^{2}$ PROFILE ABOVE DOME C}

The structure function of temperature fluctuations $D_{\theta}(\rho)$ may be computed using microthermal sensors as developed by M. Azouit and J.Vernin. ${ }^{4}$ These sensors may be fixed on a meteorological balloon in order to retrieve the $C_{n}^{2}$ profile in the atmosphere. This technic has been used during the first winterover in 2005 at Dome $\mathrm{C}$ in order to study the vertical profile of the optical turbulence. Between March 2005 and October 2005, 34 balloons were launched. Balloon profiles are resampled to a $10 \mathrm{~m}$ vertical resolution in the boundary layer (the first kilometer above the ground) and $100 \mathrm{~m}$ in the free atmosphere. The $C_{n}^{2}$ median profiles and the mean profiles of the potential temperature $\langle\theta\rangle$, mean horizontal wind speed $\left\langle V_{h}\right\rangle$ and mean direction $<\operatorname{Dir} .\left(V_{h}\right)>$ are computed over all the campaign (fig. 1). Different seasons as Autumn, Winter and Spring may be considered to study the seasonal effects on the parameters as published by Trinquet et al 2008. ${ }^{5}$

In the Boundary Layer, usually defined as the fist kilometer above the ground. The mean wind speed is less than $3 \mathrm{~ms}^{-1}$ in the first few meters and increases up to $8 \mathrm{~ms}^{-1}$ at $100 \mathrm{~m}$. The mean wind speed at the ground level may be variable from year to year, but as studied by Aristidi et al 2005, ${ }^{6}$ with a median value of $2.9 \mathrm{~ms}^{-1}$, Dome $\mathrm{C}$ is one of the lowest mean ever recorded for an observatory. Even in the case of low wind speed, the median $C_{n}^{2}$ profile shows a clear excess of optical turbulence in the first $30 \mathrm{~m}$ above the ground. The thickness of this turbulent surface layer $h_{s l}$ is defined by the part of the boundary layer containing $90 \%$ of the total boundary optical turbulence:

$$
\frac{\int_{8 m}^{h_{s l}} C_{n}^{2}(z) d z}{\int_{8 m}^{1 k m} C_{n}^{2}(z) d z}<0.9 .
$$

The first point is fixed at $8 \mathrm{~m}$, to be at the same level used for the DIMM monitor ${ }^{7}$ and to discard the turbulence very near the ground. During the 2005 campaign, $h_{s l}$ fluctuates between $10 \mathrm{~m}$ and $517 \mathrm{~m}$ (fig. 2). The median value is $33 \mathrm{~m}$ and the $75 \%$ percentile is $42 \mathrm{~m}$. The $C_{n}^{2}$ median profile may be considered as constant in the surface layer and then decreases exponentially following the relations:

$$
\begin{aligned}
8 m<h<33 m: & C_{n}^{2} \simeq 3.10^{-14} \\
33 m<h<100 m: & C_{n}^{2}(h)=2.110^{-6} h^{-5.6} \\
100 m<h<1000 m: & C_{n}^{2}(h)=2.410^{-16} h^{-0.7}
\end{aligned}
$$

In the free atmosphere, the median $C_{n}^{2}$ profile is quite similar than for mid-latitude sites. An excess of optical turbulence is observed above $20 \mathrm{~km}$ but only 3 flights reached this altitude. Most of the time the balloons 

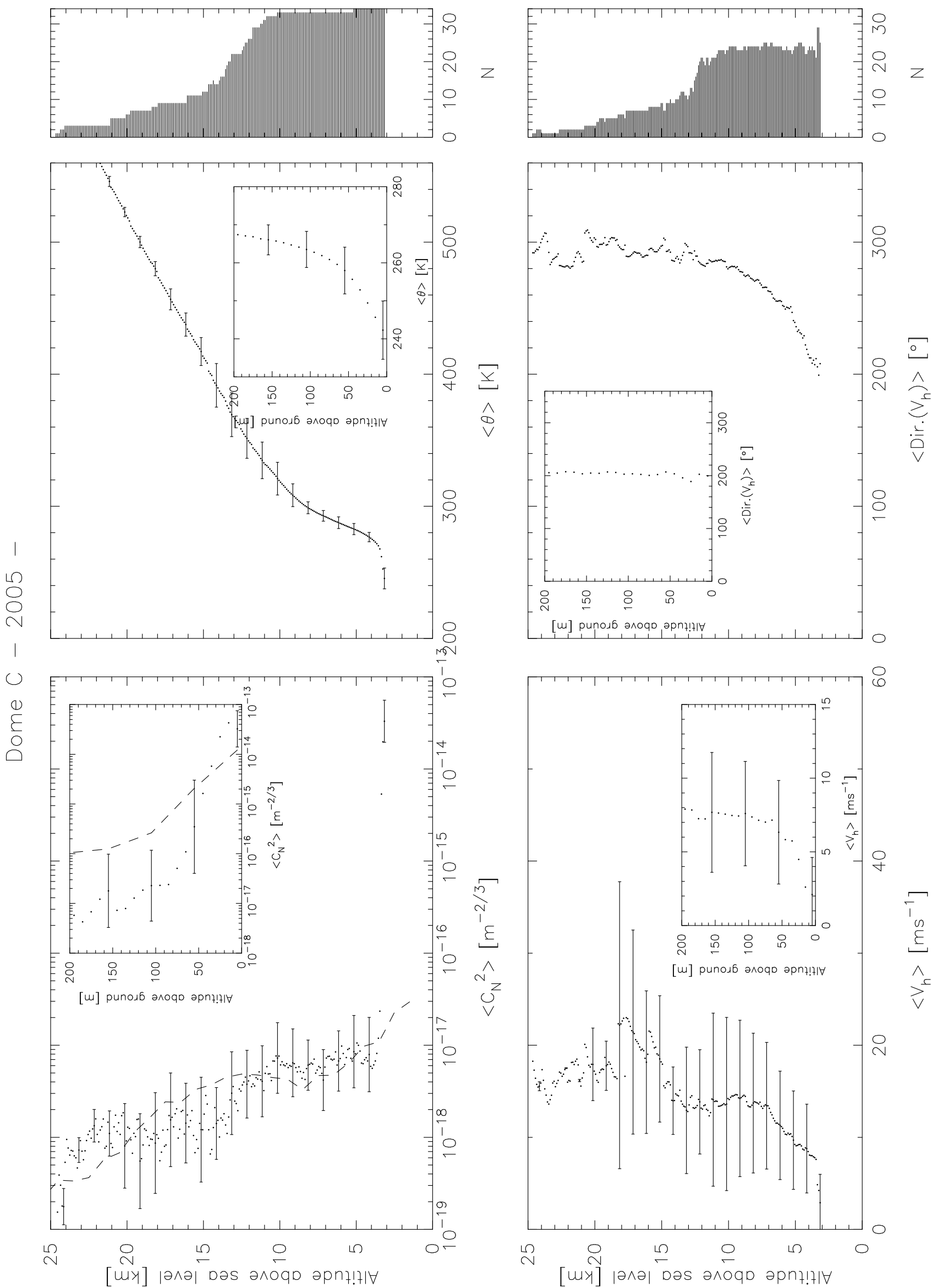

Figure 1. Median $\left\langle C_{n}^{2}\right\rangle$, mean potential temperature $\langle\theta\rangle$, mean horizontal wind speed $\left\langle V_{h}\right\rangle$ and mean wind direction $<$ Dir $>$ profiles (dots), computed from the balloons launched from the 34 balloon profiles launched during the 2005 at Dome C. Standard deviations (error bars) for the $\left\langle C_{n}^{2}>\right.$ (in logarithmic scale), $<\theta>$ and $<V_{h}>$. $N$ is the number of valid flights at the corresponding altitude. The $C_{n}^{2}$ dashed curve is the median profile for mid-latitude sites. In the main plot starting at $3250 \mathrm{~m}$ over the sea level, the mean value is computed over a range of $100 \mathrm{~m}$ every $100 \mathrm{~m}$. In the subplot, starting at $5 \mathrm{~m}$ over the ground, the mean value is computed over a range of $10 \mathrm{~m}$ every $10 \mathrm{~m}$. 


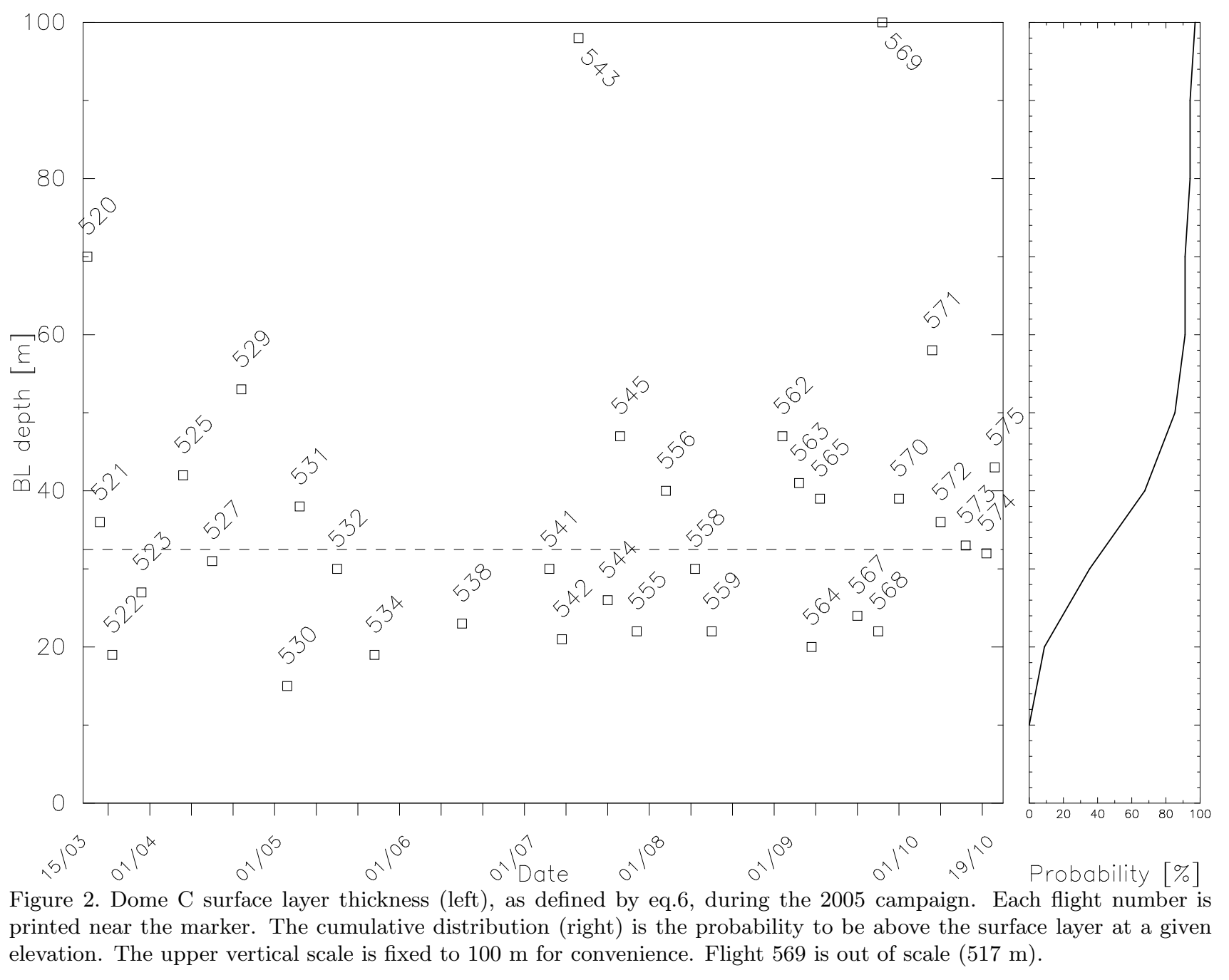

exploded when the temperature decreases below $-70^{\circ} \mathrm{C}$. The mean wind speed is particularly low when compared with mid-latitude sites. At Dome C, up to $14 \mathrm{~km}$ above sea level, the mean wind speed is below $20 \mathrm{~ms}^{-1}$. Above, the mean wind speed increases significantly in winter as quoted by Geissler and Masciadri 2006. ${ }^{8}$ This acceleration contributes to decrease the mean coherence time from $8 \mathrm{~ms}$ in Autumn to $5 \mathrm{~ms}$ in Winter. ${ }^{5}$

\section{OUTER SCALE PROFILE AT DOME C}

The outer scale of the turbulence $L_{0}$ defined the upper bound of the Kolmogorov inertial subrange in which the turbulence is fully developed, homogeneous and isotropic. In this range a systematic difference of a passive and conservative scalar as $\theta$ does not affect the amplitude of fluctuations $D_{\theta}(\rho)>>\Delta \bar{\theta}^{2}$. Tatarski in $1961^{9}$ defined the outer scale $L_{0 \mathcal{T}}$ the distance over which the field of fluctuations of $\theta$ cannot be considered locally isotropic and the fluctuational difference is no more negligible:

$$
C_{\theta}^{2} L_{0 \mathcal{T}}^{2 / 3}=a^{2}(\operatorname{grad} \bar{\theta})^{2} L_{0 \mathcal{T}}^{2},
$$

where $a$ is a constant $\left(a^{2} \approx 2.8\right)$. According to this expression, the outer scale $L_{0 \mathcal{T}}$ as defined by Tatarski may be deduced from $C_{\theta}^{2}$ profiles and $\theta$ profiles. From the Dome $\mathrm{C}$ measurements we estimate $L_{0 \mathcal{T}}$ for each profiles and then the median profiles (fig. 3). From the median profile it is clear that most of the time $L_{0 \mathcal{T}}$ is smaller than $1 \mathrm{~m}$ and smaller than outer scales usually retrieved from other sites. In the boundary layer, from the ground up 

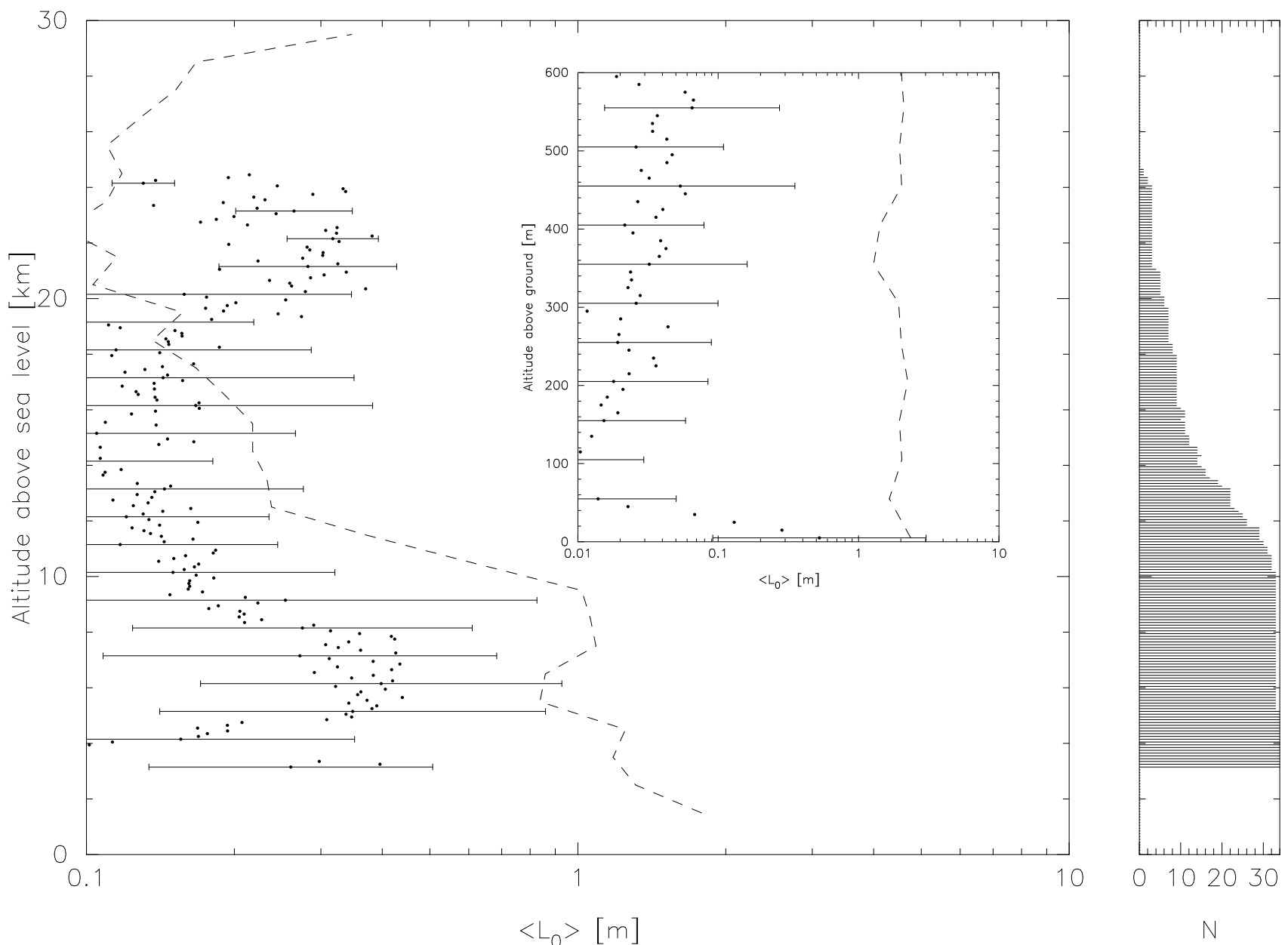

Figure 3. Median profile of the Tatarski outer scale $L_{0 \mathcal{T}}$, as defined by equation 11 , computed from the $2005 \mathrm{Dome} \mathrm{C}$ campaign. The dashed curve is the median profile for mid-latitude sites.

to $100 \mathrm{~m}, L_{0 \mathcal{T}}$ decreases rapidly from $1 \mathrm{~m}$ to few centimeters. In the free atmosphere two main slabs with large $L_{0 \mathcal{T}}$ are noticeable, in the troposphere between $5 \mathrm{~km}$ to $8 \mathrm{~km}$, and in the stratosphere above $20 \mathrm{~km}$. These two slabs are at the same levels than the turbulent slabs observed in the $C_{n}^{2}$ median profile.

\section{SPATIAL RESOLUTION AT DOME C}

The contribution of the optical turbulence on the wave front is described by a set of spatial, angular and temporal parameters. The atmospheric diffraction-limited equivalent pupil $r_{0}$, as defined by Fried 1966, is related to the $C_{n}^{2}$ profile for a plane wave crossing the atmosphere along the zenith angle $\gamma$ and at the wave length $\lambda$ :

$$
r_{0}^{-5 / 3}=16.7 \lambda^{-2} \cos (\gamma)^{-1} \int_{0}^{\infty} C_{n}^{2}(h) d h .
$$

The resolving power of a telescope of diameter $D$ is limited by $D$ when $r_{0}<D$ and is limited by $r_{0}$ when $r_{0}>D$. The seeing, the angular resolving power of the telescope for a long exposure time, considering the turbulent wave, is related to the Fried parameter $r_{0}$ by:

$$
r_{0}=0.98 \lambda / \epsilon_{0} .
$$


The isoplanatic angle $\theta_{0}$, as defined by Roddier et al 1982, is the angular domain in which phase fluctuations induced by optical turbulence are still correlated:

$$
\theta_{0}=0.058 \lambda^{6 / 5}\left[\int_{0}^{\infty} h^{5 / 3} C_{n}^{2}(h) d h\right]^{-3 / 5} .
$$

$\theta_{0}$ is more sensitive to high turbulent layers and it is roughly the field of view in which the adaptive optics remains efficient. Temporal properties of the turbulent wave front may be quantified by the coherence time $\tau_{0}$. It is the time during which the phase fluctuations are still correlated. $\tau_{0}$ is function of the $C_{n}^{2}$ profile and of the horizontal wind speed $V$ of the turbulent layers:

$$
\tau_{0}=0.058 \lambda^{6 / 5}\left[\int_{0}^{\infty}|V(h)|^{5 / 3} C_{n}^{2}(h) d h\right]^{-3 / 5} .
$$

$\tau_{0}$ sets the upper limit of adaptive optics bandwidth. Effects of the optical turbulence over large pupil diameter or interferometers have been studied by Borgnino $1990^{10}$ which defined the spatial-coherence outer scale $\mathscr{L}_{0}$. The first definition used the Tatarski outer-scale $L_{0 \mathcal{T}}$ in order to limit the low spatial frequencies of the phase fluctuations:

$$
\mathscr{L}_{0}^{-1 / 3}=\frac{\int_{0}^{\infty} L_{0}(h)^{-1 / 3} C_{n}^{2}(h) d h}{\int_{0}^{\infty} C_{n}^{2}(h) d h},
$$

so that the variance of the beam at the focus of a telescope of diameter $D$ is:

$$
\sigma^{2} \approx 0.18 \lambda^{2} r_{0}^{-5 / 3}\left[D^{-1 / 3}-\mathscr{L}_{0}^{-1 / 3}\right] .
$$

In a second publication, Borgnino 1992 considered the von Kármán spectra as a relevant expression to describe the saturation of the phase structure function out of the inertial range $\left(\rho>L_{0}\right)$ and gives a second definition of the spatial-coherence outer scale $\mathscr{L}_{0 \mathcal{V}_{K}}$ using the von Kármán outer scale $L_{0 \mathcal{V}_{K}}$. The structure function for temperature fluctuations is then :

$$
D_{\theta}(\rho)=\int_{0}^{\infty}[1-\operatorname{sinc}(k r)] \Phi_{\theta}(k) k^{2} d k
$$

where $\Phi_{T}(k)$ is the three-dimensional temperature fluctuations spectrum:

$$
\Phi_{\theta}(k)=0.033 C_{\theta}^{2}\left[k^{2}+\left(\frac{2 \pi}{L_{0 \mathcal{V} K}}\right)^{2}\right]^{-11 / 6} .
$$

One has to pay attention on the fact that the outer scale defined by von Kármán $L_{0 \mathcal{V} K}$ is different of the Tatarski $L_{0 \mathcal{T}}$. Tatarski considered the intersection of the two asymptotes $D_{\theta}\left(\rho<<L_{0}\right)$ and $D_{\theta}\left(\rho>>L_{0}\right)$ :

$$
D_{\theta}\left(L_{0_{\mathcal{T}}}\right)=a C_{\theta}^{2} L_{0 \mathcal{T}}^{2 / 3}=\Delta \theta^{2},
$$

and the von Kármán structure function has the asymptotic value $D_{\theta}\left(\rho>>L_{0}\right)$ :

$$
D_{\theta}\left(\rho>>L_{0}\right)=8 \pi \int_{0}^{\infty} K^{2} \Phi_{\theta}(k) d k=0.307 C_{\theta}^{2} L_{0 \mathcal{V} K}^{2 / 3} .
$$

So a relation between them may be deduced as:

$$
\begin{gathered}
L_{0 \mathcal{V} K}=\alpha L_{0 \mathcal{T}}, \\
\alpha=\left(\frac{a}{0.307}\right)^{3 / 2}
\end{gathered}
$$

It is clear that $L_{0 \mathcal{V} K}$ overestimates the inertial subrange as defined by Tatarski by a factor $\alpha=12.7$ in which the classical theory of the wave propagation in a turbulent media may be applied. $\alpha$ has been experimentaly 
estimated $\alpha \approx 13$ by simultaneous measurements of the spatial coherence outer scale $\mathscr{L}_{0}$ between the GSM monitor, using the von Kármán spectra, and $C_{n}^{2}$ balloon profiles using the Tatarski definition. ${ }^{11} \mathrm{~A}$ factor has to be introduced in the von Kármán spectra so that the same outer scale $L_{0 \mathcal{T}}$ may be considered as published by Reinhardt in $1972^{12}$ and focused by Greenwood and Tarazano in 2008: ${ }^{13}$

$$
\Phi_{\theta}(k)=0.033 C_{\theta}^{2}\left[k^{2}+\left(1.071 \frac{2 \pi}{L_{0 \mathcal{T}}}\right)^{2}\right]^{-11 / 6} .
$$

Table 1 gives the statistical values of the considered integrated parameters for Dome $\mathrm{C}$ deduced from the balloon profiles.

Table 1. Statistics of the integrated wave front parameters as deduced from the balloon profiles of the 2005 Dome C campaign. Median values and quartiles $\left(\begin{array}{c}q_{+} \\ q_{-}\end{array}\right)$are given. Two different elevations above the ground $h_{0}$ are considered. As only 3 flights are available to estimate $\theta_{0}$ with less than $10 \%$ of error, only the $\theta_{0}$ median value is given.

\begin{tabular}{l|ccccc}
$h_{0}$ & $\epsilon_{0}$ & $\theta_{0}$ & $\tau_{0}$ & $\mathscr{L}_{0 \mathcal{T}}$ & $\mathscr{L}_{0 \mathcal{V} K}$ \\
{$[m]$} & {$\left[{ }^{\prime \prime}\right]$} & $\left.{ }^{\prime \prime}\right]$ & {$[m s]$} & {$[m]$} & {$[m]$} \\
\hline 8 & $1.4\left(\begin{array}{l}1 \\
1.6 \\
0.3 \\
0.6\end{array}\right)$ & 2.7 & $5.7\left(\begin{array}{c}3 \\
9\end{array}\right)$ & $0.6\left(\begin{array}{c}0.4 \\
1.0\end{array}\right)$ & $7.8\left(\begin{array}{c}4.6 \\
13.0\end{array}\right)$ \\
33 & 0.4 & $6.8\left(\begin{array}{c}5 \\
12\end{array}\right)$ & $0.3\left(\begin{array}{c}0.2 \\
0.4\end{array}\right)$ & $3.2\left(\begin{array}{c}2.3 \\
5.1\end{array}\right)$ \\
\hline
\end{tabular}

\section{CONCLUSIONS}

Dome $\mathrm{C}$ is noticeable by the very strong and thin optical turbulent surface layer. To improve significantly the seeing conditions, telescopes at Dome $\mathrm{C}$ should be elevated over the turbulent surface layer. Another strategy could be to develop a specific adaptive optic. As the surface layer is so thin, a single adaptive optic system should be able to improve and damp significantly the seeing over a large field of view. The free atmosphere turbulence is also specific at Dome $\mathrm{C}$ with small outer scales and no main turbulent layers. Thin turbulent layers occurred in two turbulent slabs in the troposphere between $5 \mathrm{~km}$ to $8 \mathrm{~km}$ and in the mid-stratosphere above $20 \mathrm{~km}$. The mean wind speed is particularly low in the troposphere and during the beginning of the winter. This explain the large mean value of coherence time. As the upper part of the atmosphere has been sensed only during the beginning of the winter, a better statistics should be provided by the Single Star Scidar SSS from the 2006 campaign.

\section{ACKNOWLEDGMENTS}

The authors wish to thank all the people who have contributed to this campaign, the summer camp logistics and the winterover team of Concordia. This program has been supported by the polar institutes IPEV and PNRA. We thank also the Association of Universities for Research in Astronomy (AURA) for funding the Vaisala station and the US Air Force (contract F61775-02-C002) for part of the microthermal payloads. Part of this work has also been funded by the French Agence Nationale de la Recherche under contract title "CASDOA" (ANR-05BLAN-0033-01).

\section{REFERENCES}

[1] Richardson, L., [Weather Prediction by Numerical Process], Cambridge University Press (1922).

[2] Kolmogorov, A., "The Local Structure of Turbulence in Incompressible Viscous Fluid for Very Large Reynolds' Numbers," Comptes Rendus de l'Academie des Sciences de l'URSS 30, 301-305 (1941).

[3] Obukhov, A., "Structure of the Temperature Field in a Turbulent Flow," Bulletin of the Academy of Sciences of the USSR, Geographical and Geophysicla Series 13, 58-69 (1949).

[4] Azouit, M. and Vernin, J., "Optical Turbulence Profiling with Balloons Relevant to Astronomy and Atmospheric Physics," PASP 117, 536-543 (May 2005). 
[5] Trinquet, H., Agabi, A., Vernin, J., Azouit, M., Aristidi, E., and Fossat, E., "Nighttime Optical Turbulence Vertical Structure above Dome C in Antarctica," PASP 120, 203-211 (Feb. 2008).

[6] Aristidi, E., Agabi, K., Azouit, M., Fossat, E., Vernin, J., Travouillon, T., Lawrence, J. S., Meyer, C., Storey, J. W. V., Halter, B., Roth, W. L., and Walden, V., "An analysis of temperatures and wind speeds above Dome C, Antarctica," A\&BA 430, 739-746 (Feb. 2005).

[7] Aristidi, E., Agabi, A., Fossat, E., Azouit, M., Martin, F., Sadibekova, T., Travouillon, T., Vernin, J., and Ziad, A., "Site testing in summer at Dome C, Antarctica," A $\& A$ 444, 651-659 (Dec. 2005).

[8] Geissler, K. and Masciadri, E., "Meteorological Parameter Analysis above Dome C Using Data from the European Centre for Medium-Range Weather Forecasts," PASP 118, 1048-1065 (July 2006).

[9] Tatarski, V., [Wave Propagation in a Turbulent Medium], New-York (1961).

[10] Borgnino, J., "Estimation of the spatial coherence outer scale relevant to long baseline interferometry and imaging in optical astronomy," Appl. Opt. 29, 1863-1865 (May 1990).

[11] Abahamid, A., Jabiri, A., Vernin, J., Benkhaldoun, Z., Azouit, M., and Agabi, A., "Optical turbulence modeling in the boundary layer and free atmosphere using instrumented meteorological balloons," $A \mathscr{E} A$ 416, 1193-1200 (Mar. 2004).

[12] Reinhardt, G. and Collins, S., "Outer-Scale Effects in Turbulence-Degraded Light Beam Spectra," J. Opt. Soc. Am. 62, 1526-1530 (1972).

[13] Greenwood, D. and Tarazano, D., "Proposed form for the atmospheric turbulence spatial spectrum at large scales," J. Opt. Soc. Am. 25, 1349-1360 (May 2008). 